\title{
Global Optimization using a Dynamical Systems Approach
}

\author{
Stefan Sertl and Michael Dellnitz* \\ Faculty of Computer Science, \\ Electrical Engineering and Mathematics \\ University of Paderborn \\ D-33095 Paderborn \\ http://math-www. uni-paderborn.de/ agdellnitz
}

July 21, 2003

\begin{abstract}
We develop new algorithms for global optimization by combining well known branch and bound methods with multilevel subdivision techniques for the computation of invariant sets of dynamical systems. The basic idea is to view iteration schemes for local optimization problems - e.g. Newton's method or conjugate gradient methods - as dynamical systems and to compute set coverings of their fixed points. The combination with bounding techniques allow for the computation of coverings of the global optima only. We show convergence of the new algorithms and present a particular implementation.
\end{abstract}

Key words: global optimization, branch and bound optimization, dynamical systems, computation of fixed points

MSC2000 - Classification: 37N40, 65K05, 90C30

\footnotetext{
* Research of the authors is partially supported by the Deutsche Forschungsgemeinschaft within the Sonderforschungsbereich 376.
} 


\section{Introduction}

The solution of global optimization problems plays an important role in many applications in science and engineering. Classical iteration procedures (e.g. Newton's method or conjugate gradient methods) are often not appropriate for finding a global minimum of a given objective function, since they usually get stuck in local minima. Alternative approaches like random search methods (see e.g. Dixon and Szegö (1978), Zhigljavsky (1991)) or genetic algorithms (Goldberg (1989); Davis (1996)) have the disadvantage that there always is some uncertainty whether or not the global optimum has actually been found at the end of the searching procedure. On the other hand rigorous methods based on interval analysis as proposed for instance in Ratschek and Rokne (1988), Hansen (1992) or Kearfott (1996) are usually only applicable to low-dimensional problems.

In this article we propose a new method for the computation of the global minimum of a given objective function. This method is based on a set oriented approach which is similar in spirit as the one in Dellnitz and Hohmann (1997) where set oriented numerical methods have been developed for the analysis of discrete dynamical systems (see also Dellnitz and Hohmann (1996); Dellnitz and Junge (2002)). Roughly speaking these methods work as follows: starting with a big compact region in state space one constructs successively refined box coverings of the invariant sets of the dynamical system under consideration. Thus, the respective numerical multilevel scheme is based on two basic ingredients: subdivision and selection. Similar approaches can be found e.g. in Hsu (1987) or Osipenko and Komarchev (1995).

Already in Dellnitz et al. (2002) subdivision techniques were succesfully introduced for the computation of all the zeros of a nonlinear function in a compact domain. The underlying idea is to view iteration schemes (such as Newton's method) as dynamical systems. Then the subdivision and selection procedures are adapted to this context such that all the respective fixed points can be detected. In this paper we go even one step further and adjust the algorithms to the context of global optimization. That is, by an additional combination with branch and bound strategies we propose particular subdivision schemes which allow to approximate the global minima of a given function in a reliable way.

A more detailed outline of the article is as follows. In Section 2 we present a modification of the subdivision procedure for the computation of set coverings of the fixed points of a given dynamical system (Proposition 2.2 and Algorithm 2.1). This allows us to compute all the local extremal points of arbitrary nonlinear functions within compact domains. Since we want to find the global optima of the given objective function we propose an algorithm which combines the subdivision procedure of Section 2 with branch and bound techniques as described in Horst and Tuy (1996) and discuss its convergence properties (Section 3). In Section 4 we present three numerical examples which illustrate the efficiency and reliability of our approach. 


\section{The Computation of Fixed Points of Discrete Dynamical Systems}

We consider discrete dynamical systems of the type

$$
x_{j+1}=f\left(x_{j}\right), \quad j=0,1,2, \ldots,
$$

where $f: \mathbb{R}^{n} \rightarrow \mathbb{R}^{n}$ is continuous. Our purpose is to develop a set oriented numerical method for the approximation of all the fixed points of $f$ within some given compact subset $Q \subset \mathbb{R}^{n}$, i.e. the set

$$
F P_{f}(Q)=\{x \in Q: f(x)=x\} .
$$

The main idea is to adapt a subdivision technique used in Dellnitz and Hohmann (1997) for the approximation of general invariant sets of dynamical systems in order to compute successively finer coverings of $F P_{f}(Q)$.

\subsection{The Subdivision Procedure}

The following algorithm is a modified version of subdivision schemes, which have previously been presented in Dellnitz and Hohmann (1997). The main difference is in the selection step, which is now adapted to the purpose of finding $F P_{f}(Q)$.

Algorithm 2.1 Let $\mathcal{B}_{0}$ be an initial collection of finitely many subsets of the compact set $Q$ such that $\cup_{B \in \mathcal{B}_{0}} B=Q$. Then $\mathcal{B}_{k}$ is inductively obtained from $\mathcal{B}_{k-1}$ in two steps:

(i) Subdivision Construct a new system $\widehat{\mathcal{B}}_{k}$ of subsets such that

$$
\bigcup_{B \in \widehat{\mathcal{B}}_{k}} B=\bigcup_{B \in \mathcal{B}_{k-1}} B
$$

and

$$
\operatorname{diam}\left(\widehat{\mathcal{B}}_{k}\right)=\theta_{k} \operatorname{diam}\left(\mathcal{B}_{k-1}\right),
$$

where $0<\theta_{\min } \leq \theta_{k} \leq \theta_{\max }<1$.

(ii) Selection Define the new collection $\mathcal{B}_{k}$ by

$$
\mathcal{B}_{k}=\left\{B \in \widehat{\mathcal{B}}_{k}: f(B) \cap B \neq \emptyset\right\}
$$

As a first result we show that this algorithm converges to the set $F P_{f}(Q)$ for $k \rightarrow \infty$.

Proposition 2.2 Let $Q_{k}$ be the union of the subsets in $\mathcal{B}_{k}$,

$$
Q_{k}=\bigcup_{B \in \mathcal{B}_{k}} B
$$

Then the following holds: 
(a) $Q_{k}$ is a covering of $F_{f}(Q)$ for every $k \geq 0$.

(b) $Q_{\infty}=\bigcap_{k=0}^{\infty} Q_{k}=F P_{f}(Q)$.

Proof:

(a) Let $x \in F P_{f}(Q)$. By definition we have $x \in Q=Q_{0}$. Now assume that $x \in Q_{k-1}$ for some $k>0$. Then by construction there exists a $B(x) \in \widehat{\mathcal{B}}_{k}$ with $x \in B(x)$. Since $f(x)=x$ it follows that $f(B(x)) \cap B(x)$ is nonempty. Therefore $B(x) \in \mathcal{B}_{k}$ which implies $x \in Q_{k}$.

(b) Let $x \in Q_{\infty}$. Then for every $k \geq 0$ there exists a $B_{k}(x) \in \mathcal{B}_{k}$ with $x \in B_{k}(x)$. Assume that $x \notin F P_{f}(Q)$, i.e. $\varepsilon=\|f(x)-x\|>0$. Since $f$ is continuous there exists a $\delta>0$ such that $\left\|f\left(x^{\prime}\right)-f(x)\right\|<\varepsilon / 3$ for every $x^{\prime} \in \mathbb{R}^{n}$ with $\left\|x^{\prime}-x\right\|<\delta$. Using the fact that $\operatorname{diam}\left(\mathcal{B}_{k}\right) \rightarrow 0$ for $k \rightarrow \infty$ we conclude that there exists a $K>0$ such that $\operatorname{diam}\left(B_{k}(x)\right)<\min (\delta, \varepsilon / 3)$ for all $k>K$. But this implies that $\operatorname{diam}\left(f\left(B_{k}(x)\right)\right)<2 \varepsilon / 3$ for all $k>K$. On the other hand $f(B) \cap B \neq \emptyset$ for all $B \in \mathcal{B}_{k}$ and all $k>0$, thus we have a contradiction. We conclude that $Q_{\infty} \subset F P_{f}(Q)$. In combination with (a) it immediately follows that $Q_{\infty}=F P_{f}(Q)$.

Remark 2.3 The statements of Proposition 2.2 still hold if we replace $f(B)$ in the selection step of the subdivision procedure with some outer approximation $U(f(B))$ of $f(B)$ as long as $\max _{B \in \widehat{\mathcal{B}}_{k}} \operatorname{dist}(U(f(B)), f(B)) \rightarrow 0$ for $k \rightarrow \infty$.

\subsection{Implementation of the Algorithm}

The numerical realization of the subdivision procedure is very similar to the classical subdivision algorithm for the approximation of arbitrary invariant sets of dynamical systems as described in Dellnitz and Hohmann (1997). For the sake of completeness we briefly review its important aspects.

For the implementation of the collections $\mathcal{B}_{k}$ we use generalized rectangles (also called boxes) of the form

$$
R(c, r)=\left\{y \in \mathbb{R}^{n}:\left|y_{i}-c_{i}\right| \leq r_{i} \text { for } i=1, \ldots, n\right\},
$$

where $c, r \in \mathbb{R}^{n}, r_{i}>0$ for $i=1, \ldots, n$, are the center and radius respectively. We start the subdivision procedure with a single rectangle $\mathcal{B}_{0}=\{R\}$. Given a collection $\mathcal{B}_{k}$ we construct the refined collection $\widehat{\mathcal{B}}_{k}$ by bisection of the rectangles in $\mathcal{B}_{k}$ with respect to the $j$-th coordinate, where $j$ is varied cyclically. The subdivision of a rectangle $R(c, r)$ leads to two rectangles $R_{-}\left(c^{-}, \hat{r}\right)$ and $R_{+}\left(c^{+}, \hat{r}\right)$, where

$$
\hat{r}_{i}=\left\{\begin{array}{cl}
r_{i} & \text { for } i \neq j \\
r_{i} / 2 & \text { for } i=j
\end{array}, \quad c_{i}^{ \pm}=\left\{\begin{array}{cc}
c_{i} & \text { for } i \neq j \\
c_{i} \pm r_{i} / 2 & \text { for } i=j
\end{array} .\right.\right.
$$




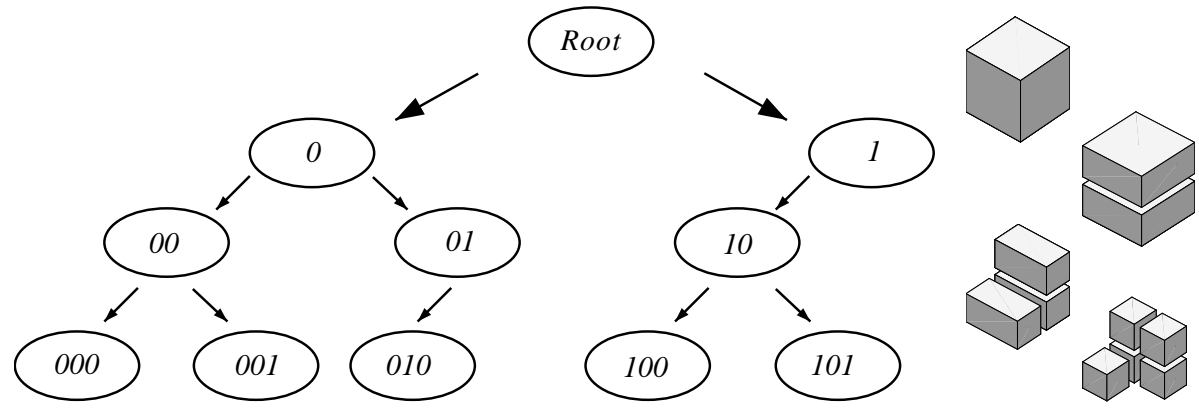

Figure 1: Storage scheme for the box collections $\mathcal{B}_{k}, k=0,1,2,3$.

This allows for a very efficient storage scheme: a collection $\mathcal{B}_{k}$ is completely determined by the initial box $R(c, r)$ and a binary tree representing the subdivision structure, see Figure 1.

The selection step is usually discretized via test points within each box. The selection criteria is thus given by

$$
\mathcal{B}_{k}=\left\{B \in \widehat{\mathcal{B}}_{k}: f(x) \in B \text { for at least one test point } x \in B\right\} .
$$

For low-dimensional problems we typically use a fixed grid of test points within each box, in higher-dimensional problems the points are chosen at random.

REMARK 2.4 Rigorous convergence results for the realization of the subdivision scheme can be obtained when outer approximations of $f(B)$ can be computed which satisfy the condition of Remark 2.3. This can be done for example using appropriate interval extensions of $f$ (see e.g. Moore (1966); Alefeld and Herzberger (1983)). If local Lipschitz estimates on $f$ are available the methods presented in Junge (1999) can be used, too.

We now illustrate the method by the following elementary

EXAMPLE 2.5 We want to compute the fixed points of the Hénon map (Hénon (1976))

$$
f: \mathbb{R}^{2} \rightarrow \mathbb{R}^{2}, \quad f(x)=\left(\begin{array}{c}
1-a x_{1}^{2}+b x_{2} \\
x_{1}
\end{array}\right)
$$

with parameters $a=1.2$ and $b=0.2$.

Using Algorithm 2.1 with a grid of $4 \times 4$ test points per box in the selection step we get the coverings shown in Figure 2. After 40 subdivision steps the covering consists of 5 boxes within two clusters (see Table I).

Since the number of clusters stays constant after the first few subdivision steps we conclude that there are two fixed points within the rectangles

$$
[-1.3051645,-1.3051528] \times[-1.3051645,-1.3051528]
$$




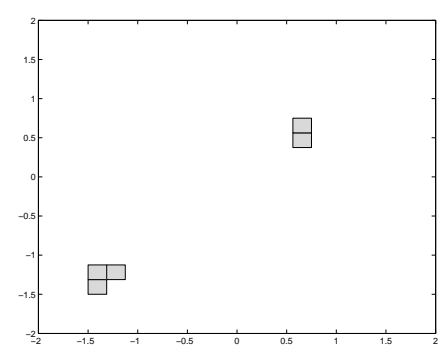

(a) $k=10$

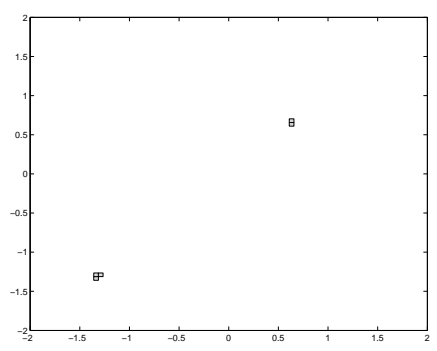

(b) $k=14$

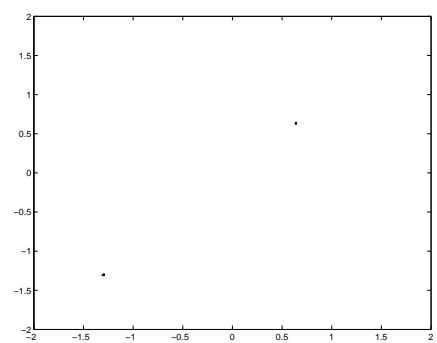

(c) $k=18$

Figure 2: Box coverings $\mathcal{B}_{10}, \mathcal{B}_{14}$ and $\mathcal{B}_{18}$ of the fixed points of the Hénon map.

Table I: Boxes obtained after 40 steps of Algorithm 2.1 applied to the Hénon map.

\begin{tabular}{rrcc}
\hline \multicolumn{2}{c}{ center } & \multicolumn{2}{c}{ radius } \\
\hline$x_{1}$ & $x_{2}$ & $x_{1}$ & $x_{2}$ \\
\hline-1.30516148 & -1.30516148 & $2.86102295 \cdot 10^{-6}$ & $2.86102295 \cdot 10^{-6}$ \\
-1.30515575 & -1.30516148 & $2.86102295 \cdot 10^{-6}$ & $2.86102295 \cdot 10^{-6}$ \\
-1.30515575 & -1.30515575 & $2.86102295 \cdot 10^{-6}$ & $2.86102295 \cdot 10^{-6}$ \\
\hline 0.63849163 & 0.63849163 & $2.86102295 \cdot 10^{-6}$ & $2.86102295 \cdot 10^{-6}$ \\
0.63849163 & 0.63849735 & $2.86102295 \cdot 10^{-6}$ & $2.86102295 \cdot 10^{-6}$ \\
\hline
\end{tabular}

and

$$
[0.6384887,0.6384945] \times[0.6384887,0.6385003]
$$

respectively. The exact results can either be found by additional subdivision steps until a prescribed accuracy is achieved or by switching to some local search method which uses the obtained covering as input.

\section{Application to Optimization Problems}

In this section we show how to use the methods presented in the previous section to solve unconstraint optimization problems.

\subsection{Computation of Extremal Points}

The main idea is to view iteration schemes for local optimization (e.g. Newton's method or conjugate gradient methods) as discrete dynamical systems and to compute their fixed points using the subdivision procedure of Section 2. Since these fixed points correspond to the (local) extremal solutions of the objective function under consideration this method allows for the computation of coverings of all the minima (or maxima) within a given compact subset of the phase space. 
EXAMPLE 3.1 We want to compute the extremal points of the following objective function (cf. Himmelblau (1972)):

$$
g\left(x_{1}, x_{2}\right)=\left(x_{1}^{2}+x_{2}-11\right)^{2}+\left(x_{1}+x_{2}^{2}-7\right)^{2}
$$

Within the compact set $Q=[-5,5] \times[-5,5]$ this function possesses 9 critical points as listed in Table II. Using our subdivision algorithm with Newton's method as the dynamical system and a grid of $3 \times 3$ test points per box we obtain the covering shown in Figure 3. It consists of 9 boxes with each of them containing one of the critical points of $g$.

Table II: Locations and types of the critical points of $g$ within the region $Q=$ $[-5,5] \times[-5,5]$.

\begin{tabular}{rrc}
\hline$x_{1}$ & \multicolumn{1}{c}{$x_{2}$} & type \\
\hline-3.7793 & -3.2832 & minimum \\
-2.8051 & 3.1313 & minimum \\
3.0000 & 2.0000 & minimum \\
3.5844 & -1.8481 & minimum \\
-3.0730 & -0.0814 & saddle point \\
-0.1280 & -1.9537 & saddle point \\
0.0867 & 2.8843 & saddle point \\
3.3852 & 0.0739 & saddle point \\
-0.2708 & -0.9230 & maximum \\
\hline
\end{tabular}

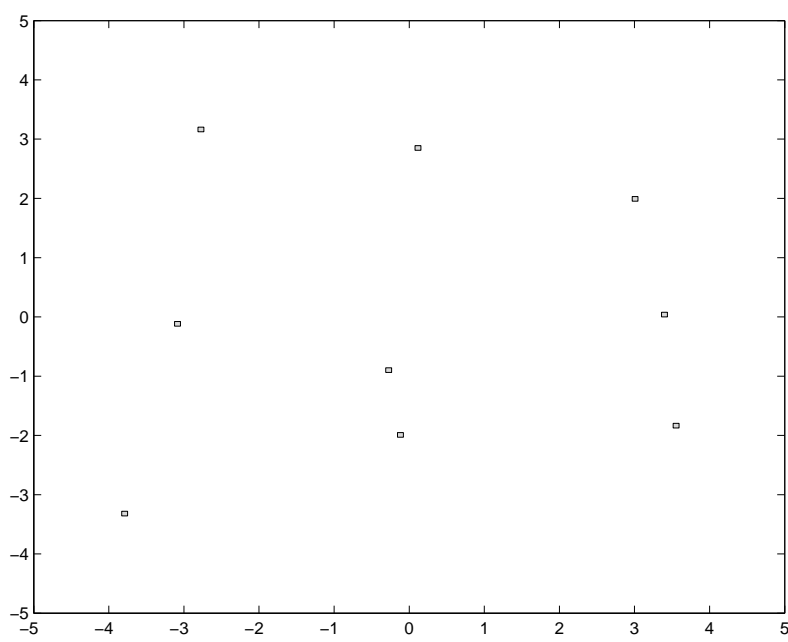

Figure 3: Box covering of all the critical points of the function $g$ of Example 3.1 obtained after 14 subdivision steps. 
Having computed such a box covering one can obtain the exact solutions of the optimization problem using standard iterative optimization algorithms with a small number of initial points within each of the boxes.

\subsection{Combination with Branch and Bound Techniques}

Using the method described above we are in principle able to approximate all the local extremal solutions of the given optimization problem. Selection of the global minima (or maxima) can then be done by comparing the corresponding function values.

However, if one is only interested in the global minima our method can be combined with well known branch and bound techniques (see e.g. Horst and Tuy (1996)) yielding an even more efficient approach. We therefore introduce the following modified algorithm for the computation of coverings of the set of global minimizers of a given continuous function $g: \mathbb{R}^{n} \rightarrow \mathbb{R}$ :

Algorithm 3.2 Let $f: \mathbb{R}^{n} \rightarrow \mathbb{R}^{n}$ be an iteration scheme such that the local minima of $g$ are fixed points of $f$. Set $\alpha_{0}=+\infty$ and let $\mathcal{B}_{0}$ be an initial collection of finitely many subsets of a compact set $Q$ such that $\cup_{B \in \mathcal{B}_{0}} B=Q$. Then $\mathcal{B}_{k}$ is inductively obtained from $\mathcal{B}_{k-1}$ in two steps:

(i) Subdivision Construct a new system $\widehat{\mathcal{B}}_{k}$ of subsets such that

$$
\bigcup_{B \in \widehat{\mathcal{B}}_{k}} B=\bigcup_{B \in \mathcal{B}_{k-1}} B
$$

and

$$
\operatorname{diam}\left(\widehat{\mathcal{B}}_{k}\right)=\theta_{k} \operatorname{diam}\left(\mathcal{B}_{k-1}\right),
$$

where $0<\theta_{\min } \leq \theta_{k} \leq \theta_{\max }<1$.

(ii) Selection For each $B \in \widehat{\mathcal{B}}_{k}$ determine a lower bound $\beta_{k}(B) \leq \inf g(B)$. Let $\alpha_{k}=\min \left(\alpha_{k-1}, \min g\left(S_{k}\right)\right)$ for a finite set of sample points $S_{k}$ within the union of the boxes in $\widehat{\mathcal{B}}_{k}$.

The new collection $\mathcal{B}_{k}$ is then defined by

$$
\mathcal{B}_{k}=\left\{B \in \widehat{\mathcal{B}}_{k}: f(B) \cap B \neq \emptyset \text { and } \beta_{k}(B) \leq \alpha_{k}\right\} .
$$

From Proposition 2.2 we know that in the limit the resulting set $Q_{\infty}=\bigcap_{k=0}^{\infty} Q_{k}$ with $Q_{k}=\cup_{B \in \mathcal{B}_{k}} B$ contains only fixed points of $f$. We now show under which conditions Algorithm 3.2 converges to the set

$$
G M_{g}(Q)=\left\{x^{\star} \in Q: g\left(x^{\star}\right) \leq g(x) \forall x \in Q\right\}
$$

of all global minimizers of $g$ with respect to the set $Q$. 
TheOREM 3.3 The set $Q_{\infty}=\bigcap_{k=0}^{\infty} Q_{k}$ with $Q_{k}=\cup_{B \in \mathcal{B}_{k}} B$ generated by Algorithm 3.2 is equal to the set $G M_{g}(Q)=\left\{x^{\star} \in Q: g\left(x^{\star}\right) \leq g(x) \forall x \in Q\right\}$ of global minimizers of $g$ with respect to $Q$ if the following conditions on the bounds $\alpha_{k}$ and $\beta_{k}(B)$ are satisfied:

(i) $\left(\alpha_{k}-\min g(Q)\right) \rightarrow 0$ for $k \rightarrow \infty$.

(ii) $\max _{B \in \mathcal{B}_{k}}\left(\inf g(B)-\beta_{k}(B)\right) \rightarrow 0$ for $k \rightarrow \infty$.

Proof: We first show that no global minimizer is removed in the selection step of the algorithm: Let $x \in Q$ be a global minimizer of $g$ with respect to $Q$. Assume that $x \in Q_{k-1}=\bigcup_{B \in \mathcal{B}_{k-1}} B$ for some $k>0$. By construction there exists a $B(x) \in \widehat{\mathcal{B}}_{k}$ with $x \in B(x)$. Since $x$ is a fixed point of $f$ we have $f(B(x)) \cap B(x) \neq \emptyset$. Furthermore $\alpha_{k} \geq g(x)$ since $x$ is a global minimizer and $g(x) \geq \beta_{k}(B(x))$ by construction. It follows that $B(x) \in \mathcal{B}_{k}$ and therefore $x \in Q_{k}$. Since $x \in Q=Q_{0}$ we conclude that $x \in Q_{k}$ for all $k \geq 0$ and hence $x \in Q_{\infty}$.

Since $Q_{\infty}$ is a subset of $F P_{f}(Q)$ by Proposition 2.2 it remains to show that each $x \in F P_{f}(Q)$ which is not a global minimizer of $g$ is not contained in $Q_{\infty}$ : Let $x \in F P_{f}(Q)$ with $\varepsilon=(g(x)-\min g(Q))>0$. For contradiction assume that $x \in Q_{\infty}$. This implies that for every $k \geq 0$ there exists a $B_{k}(x) \in \mathcal{B}_{k}$ with $x \in B_{k}(x)$. Since $g$ is continuous and $\operatorname{diam}\left(\mathcal{B}_{k}\right) \rightarrow 0$ for $k \rightarrow \infty$ there exists a $k_{1}$ with $\left(g(x)-\inf g\left(B_{k}(x)\right)\right)<\varepsilon / 3$ for all $k>k 1$. The condition on $\beta_{k}$ implies that there is a $k_{2}$ with $\left(\inf g\left(B_{k}(x)\right)-\beta_{k}\left(B_{k}(x)\right)\right)<\varepsilon / 3$ for all $k>k_{2}$. Furthermore there exist a $k_{3}$ such that $\left(\alpha_{k}-\min g(Q)\right)<\varepsilon / 3$ for all $k>k_{3}$ due to the convergence property of $\alpha_{k}$. It follows that for all $k>K=\max \left(k_{1}, k_{2}, k_{3}\right)$

$$
\begin{aligned}
\alpha_{k}-\beta_{k}\left(B_{k}(x)\right)= & \alpha_{k}-\min g(Q)+\min g(Q)-g(x)+ \\
& g(x)-\inf g\left(B_{k}(x)\right)+\inf g\left(B_{k}(x)\right)-\beta_{k}\left(B_{k}(x)\right) \\
< & \varepsilon / 3-\varepsilon+\varepsilon / 3+\varepsilon / 3=0
\end{aligned}
$$

Therefore $\alpha_{k}<\beta_{k}\left(B_{k}(x)\right)$ for all $k>K$ which contradicts the fact that $B_{k}(x) \in \mathcal{B}_{k}$ for all $k \geq 0$.

REMARK 3.4 (a) Observe that Algorithm 3.2 can be viewed as a particular realization of the general branch and bound algorithm by Horst and Tuy (1996). In fact, the additional selection criterion $f(B) \cap B \neq \emptyset$ turns out to be very useful for purposes of global optimization.

(b) Using the definitions of Horst and Tuy a bounding operation which satisfies conditions (i) and (ii) of Theorem 3.3 is called consistent.

In numerical realizations of Algorithm 3.2 the following two questions arise:

(i) How to choose the sets $S_{k}$ of sample points to satisfy the condition on the $\alpha_{k}$ ? 
(ii) How to determine suitable lower bounds $\beta_{k}(B)$ ?

One possible answer to the first question is given by the following result:

Proposition 3.5 Let the sets of sample points $S_{k}$ for the computation of $\alpha_{k}$ in Algorithm 3.2 be defined in such a way that $S_{k} \cap B \neq \emptyset$ for all $k>0$ and for all $B \in \mathcal{B}_{k}$. Furthermore assume that condition (ii) of Theorem 3.3 is satisfied. Then the coverings $\mathcal{B}_{k}$ converge to the set of global minimizers of $g$, i.e.

$$
\operatorname{dist}\left(Q_{k}, G M_{g}(Q)\right) \rightarrow 0 \text { for } k \rightarrow \infty \text {. }
$$

Proof: We have to show that $\alpha_{k} \rightarrow \min g(Q)$ for $k \rightarrow \infty$. Let $x^{\star} \in Q$ be a global minimizer of $g$, i.e. $g\left(x^{\star}\right)=\min g(Q)$, and choose an arbitrary $\varepsilon>0$. For each $k>0$ there exists a $B_{k}\left(x^{\star}\right) \in \mathcal{B}_{k}$ with $x^{\star} \in B_{k}\left(x^{\star}\right)$. Since $\operatorname{diam}\left(\mathcal{B}_{k}\right) \rightarrow 0$ for $k \rightarrow \infty$ and by continuity of $g$ we conclude that there exists a $\bar{k}>0$ such that $g(y)-g\left(x^{\star}\right)<\varepsilon$ for all $y \in B_{k}\left(x^{\star}\right)$ and all $k>\bar{k}$. By assumption we can choose $y_{k} \in S_{k}$ with $y_{k} \in B_{k}\left(x^{\star}\right)$ for all $k>0$. It follows that $g\left(y_{k}\right)-g\left(x^{\star}\right)<\varepsilon$ for all $k>\bar{k}$. By definition of $\alpha_{k}$ we therefore have $\alpha_{k}-g\left(x^{\star}\right)<\varepsilon$ for all $k>\bar{k}$.

In applications we usually discretize the selection criterion $f(B) \cap B \neq \emptyset$ of Algorithm 3.2 by mapping test points from the box $B$. We therefore just have to compute the values of the objective function for these points to satisfy the above condition. In practice we additionally compute the objective values for the image points $f(x)$ of those test points for which $f(x) \in B$ for $x \in B$. The reason is that in general these values are lower than those for the test points themselves. This leads to sequences $\left\{\alpha_{k}\right\}_{k}$ which converge faster to the global minimum and therefore boxes with 'big' lower bounds are eliminated earlier in the subdivision process.

Obviously the computation of lower bounds $\beta_{k}(B)$ on the function values of $g$ within a given box $B$ is in general a very difficult task. If the objective function is simple enough methods of interval arithmetic can be used. Otherwise estimates on lower bounds have to be computed based on numerical approximations of the given function and/or other available information on its local behavior.

\section{Numerical Examples}

We now illustrate the efficiency of our approach by three numerical examples.

EXAMPLE 4.1 As a first example we apply our global optimization algorithm to the function

$$
g\left(x_{1}, x_{2}\right)=\left(x_{1}^{2}+x_{2}-11\right)^{2}+\left(x_{1}+x_{2}^{2}-7\right)^{2}
$$

which has previously been considered in Example 3.1. The four local minimizers of $g$ (cf. Table II) have the same function value 0 and therefore all of them are also global minimizers. As shown in Table III and Figure 4 the subdivision procedure locates all these points efficiently in a reliable way. In this simple case interval arithmetic has been used for the computation of the lower bounds. 
Table III: Number of boxes obtained during an application of the subdivision process to the objective function $g$ of Example 4.1.

\begin{tabular}{cc}
\hline $\begin{array}{c}\text { subdivision } \\
\text { steps }\end{array}$ & $\begin{array}{c}\text { number } \\
\text { of boxes }\end{array}$ \\
\hline 2 & 4 \\
4 & 9 \\
6 & 5 \\
8 & 5 \\
10 & 5 \\
12 & 4 \\
14 & 4 \\
\hline
\end{tabular}

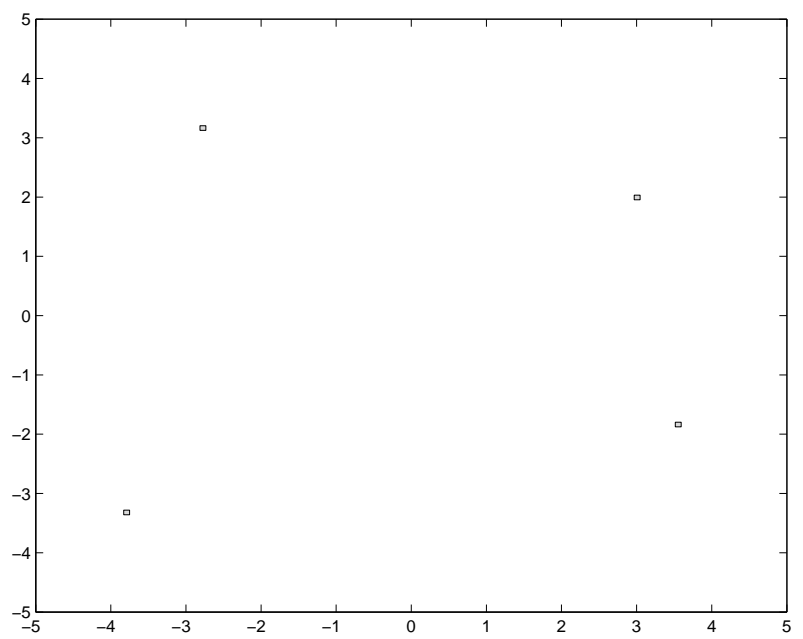

Figure 4: Box covering of the global minimizers of Example 4.1 obtained after 14 subdivision steps.

ExAmple 4.2 Our next example is taken from Moré et al. (1981):

$$
g_{2}: \mathbb{R}^{3} \rightarrow \mathbb{R}, \quad g_{2}(x)=\sum_{i=1}^{10} h_{i}(x)^{2}
$$

where

$$
h_{i}(x)=\exp \left(-0.1 i x_{1}\right)-\exp \left(-0.1 i x_{2}\right)-x_{3}(\exp (-0.1 i)+\exp (-i)) .
$$

The points $(1,10,1)^{t}$ and $(10,1,-1)^{t}$ are global minimizers. The line $x_{1}=x_{2}$, $x_{3}=0$ consists of global minimizers, too.

Using Newton's method with randomly chosen initial points the two global minima at $(1,10,1)^{t}$ and $(10,1,-1)^{t}$ can easily be found. On the other hand such a direct approach makes it difficult to detect that the line $x_{1}=x_{2}, x_{3}=0$ completely 
consists of global minimizers as well (see Figure 5(a)). In contrast to this we are able to compute coverings of all the global minimizers within a given box $B \in \mathbb{R}^{3}$ using our subdivision algorithm (cf. Figure 5(b)). As shown in Table IV a comparable computational effort is required to obtain this result. For the results in this table we have again used randomly chosen initial points for the Newton iteration.

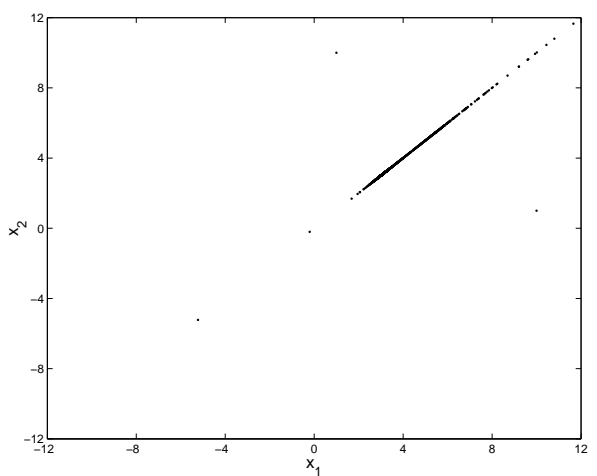

(a) Newton iteration with 25000 randomly chosen initial points

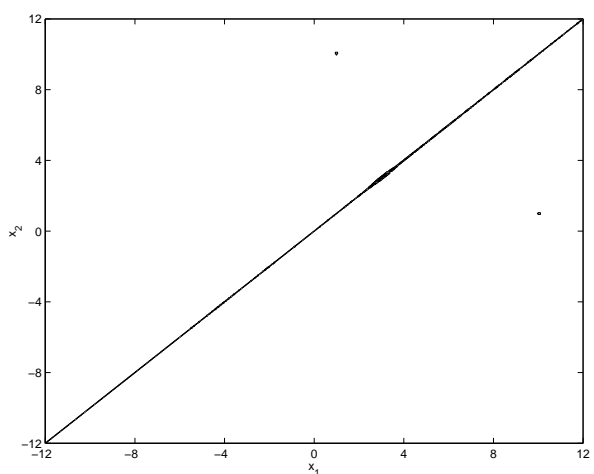

(b) Box covering after 30 steps of the subdivision algorithm

Figure 5: Global minimizers found for Example 4.2 (projection onto the $\left(x_{1}, x_{2}\right)$ plane).

Table IV: Comparison of the subdivision algorithm with other optimization methods for the function $g_{2}$.

\begin{tabular}{lcccccc}
\hline Method & \multicolumn{3}{c}{ function evaluations } & \multicolumn{3}{c}{ global minimizers found } \\
& $g_{2}$ & $\nabla g_{2}$ & $\nabla^{2} g_{2}$ & $(1,10,1)^{t}$ & $(10,1,-1)^{t}$ & $x_{1}=x_{2}$ \\
\hline Subdivision & $6.6 \cdot 10^{5}$ & $9.8 \cdot 10^{5}$ & $3.6 \cdot 10^{5}$ & yes & yes & all $^{1}$ \\
\hline Newton & 125 & $6.7 \cdot 10^{4}$ & $6.6 \cdot 10^{4}$ & yes & yes & 121 \\
& 1424 & $6.7 \cdot 10^{5}$ & $6.6 \cdot 10^{5}$ & yes & yes & 1420 \\
& 3488 & $1.7 \cdot 10^{6}$ & $1.7 \cdot 10^{6}$ & yes & yes & 3486 \\
\hline
\end{tabular}

${ }^{1}$ The line $x_{1}=x_{2}, x_{3}=0$ is completely contained in the covering.

EXAMPLE 4.3 Our last example shows that the subdivision algorithm also reliably finds the global minimizers of objective functions with a larger number of variables. For this we introduce the following function

$$
g_{3}: \mathbb{R}^{n} \rightarrow \mathbb{R}, \quad g_{3}(x)=\sum_{i=1}^{n} 1+x_{i}^{2}\left(x_{i}-0.2\right)^{2}\left(x_{i}+0.2\right)^{2}-\cos \left(10 \pi x_{i}\right)
$$

which has $3^{n}$ global minimizers in $[-2,2]^{n}$. 
Using the subdivision algorithm we always find all the global minimizers of $g_{3}$. For $n=5$ it is also possible to find all these points running the Quasi-Newton method implemented in the NAG C-library (www.nag.com) with randomly chosen initial points. However, the number of function and gradient evaluations is much higher than for the subdivision procedure (cf. Table V). For $n=10$ it seems to be impossible to compute all the minimizers using this method while the subdivision algorithm finds all of them successfully.

Table V: Comparison of the results for the subdivision algorithm and an optimization method implemented in the NAG C-library applied to the function $g_{3}$.

\begin{tabular}{|c|c|c|c|c|}
\hline \multirow[t]{2}{*}{ Dim } & \multirow[t]{2}{*}{ Method } & \multicolumn{2}{|c|}{ function evaluations } & \multirow{2}{*}{$\begin{array}{l}\text { minimizers } \\
\text { found }\end{array}$} \\
\hline & & $g_{3}$ & $\nabla g_{3}$ & \\
\hline \multirow[t]{4}{*}{5} & subdivision & $1.0 \cdot 10^{7}$ & $4.3 \cdot 10^{6}$ & 243 \\
\hline & NAG & $9.5 \cdot 10^{5}$ & $9.5 \cdot 10^{5}$ & 41 \\
\hline & & $9.6 \cdot 10^{6}$ & $9.6 \cdot 10^{6}$ & 211 \\
\hline & & $3.8 \cdot 10^{7}$ & $3.8 \cdot 10^{7}$ & 243 \\
\hline \multirow[t]{4}{*}{10} & subdivision & $1.6 \cdot 10^{10}$ & $6.7 \cdot 10^{9}$ & 59049 \\
\hline & NAG & $1.2 \cdot 10^{8}$ & $1.2 \cdot 10^{8}$ & 4 \\
\hline & & $1.2 \cdot 10^{9}$ & $1.2 \cdot 10^{9}$ & 53 \\
\hline & & $1.2 \cdot 10^{10}$ & $1.2 \cdot 10^{10}$ & 563 \\
\hline
\end{tabular}

\section{References}

G. Alefeld and J. Herzberger. Introduction to Interval Computations. Academic Press, New York, 1983.

L. Davis, editor. Handbook of Genetic Algorithms. International Thomson Computer Press, London, 1996.

M. Dellnitz and A. Hohmann. The computation of unstable manifolds using subdivision and continuation. In H.W. Broer, S.A. van Gils, I. Hoveijn, and F. Takens, editors, Nonlinear Dynamical Systems and Chaos, volume 19 of PNLDE, pages 449-459. Birkhäuser, 1996.

M. Dellnitz and A. Hohmann. A subdivision algorithm for the computation of unstable manifolds and global attractors. Numerische Mathematik, 75(3):293317, 1997.

M. Dellnitz and O. Junge. Set oriented numerical methods for dynamical systems. In N. Kopel B. Fiedler, G. Iooss, editor, Handbook of Dynamical Systems II: Towards Applications., pages 221-264. World Scientific, 2002. 
M. Dellnitz, O. Schütze, and S. Sertl. Finding zeros by multilevel subdivision techniques. IMA Journal of Numerical Analysis, 22(2):167-185, 2002.

L.C.W. Dixon and G.P. Szegö, editors. Towards Global Optimization 2. NorthHolland, Amsterdam, 1978.

D.E. Goldberg. Genetic Algorithms in Search, Optimization, and Machine Learning. Addison-Wesley, 1989.

E. Hansen. Global Optimization Using Interval Analysis. Marcel Dekker, New York, 1992.

M. Hénon. A two dimensional mapping with a strange attractor. Communications in Mathematical Physics, 50:69-77, 1976.

D.M. Himmelblau. Applied Nonlinear Programming. McGraw-Hill Book Company, New York, 1972.

R. Horst and H. Tuy. Global Optimization: Deterministic Approaches. Springer, Berlin, 3rd edition, 1996.

C.S. Hsu. Cell-to-Cell Mapping. Springer, New York, 1987.

O. Junge. Mengenorientierte Methoden zur numerischen Analyse dynamischer Systeme. PhD thesis, University of Paderborn, 1999.

B. Kearfott. Rigorous Global Search: Continuous Problems. Kluwer, Dordrecht, 1996.

R.E. Moore. Interval analysis. Prentice-Hall, Englewood Cliffs, NJ, 1966.

J. Moré, B. Garbow, and K. Hillstrom. Testing unconstrained optimization software. ACM Transactions on Mathematical Software, 7(1):17-41, 1981.

G. Osipenko and I. Komarchev. Applied symbolic dynamics: Construction of periodic trajectories. In R.P. Agarwal, editor, Dynamical systems and applications, volume 4 of Worlds Scientific Series in Applicable Analysis, pages 573-587. World Scientific, Singapore, 1995.

H. Ratschek and J. Rokne. New Computer Methods for Global Optimizations. Ellis Horwood Limited, Chichester, 1988.

A.A. Zhigljavsky. Theory of Global Random Search. Kluwer, Dordrecht, 1991. 